\title{
Probing ergodicity in granular matter
}

\author{
Fabien Paillusson and Daan Frenkel \\ Department of Chemistry, University of Cambridge, Lensfield Road, Cambridge, CB2 1EW, U.K.
}

(Dated: October 22, 2012)

\begin{abstract}
When a granular system is tapped, its volume changes. Here, using a well-defined macroscopic protocol, we prepare an ensemble of granular systems and track the statistics of volume changes as a function of the number of taps. This is in contrast to previous studies, which have focused on single trajectories and assumed ergodicity. We devise a new method to assess the convergence properties of a sequence of ensemble volume histograms and introduce a reasonable approximate version of an invariant histogram. We then compare these invariant histograms with histograms generated by sampling a long trajectory for one system and observe nonergodicity, which we quantify. Finally, we use the overlapping histogram method to assess potential compatibility with Edwards' canonical assumption. Our histograms are incompatible with this assumption.
\end{abstract}

PACS numbers: 45.70.-n,45.70.Cc,05.90.+m

\section{INTRODUCTION}

Controlling the packing density of a vibrated granular system is of industrial and scientific interest. In this paper, we focus on sequences of packings that are in mechanical equilibrium. For such packings, the effect of shaking depends solely on the tapping stage of the shaking protocol. This stage is uniquely parameterized by the extra momentum given to the system that sets the amount of dilation allowed after each tap 11.

The protocol used to prepare the granular system is also of primary importance. Currently, no macroscopic preparation protocol exists that allows one to choose the initial microscopic configuration exactly. Due to the inevitable dispersion in the initial conditions, the only reproducible quantity that one can measure in experiments is the probability of observing a given volume after a given number of taps.

During the last twenty years, properties of the time averages of packing volumes and their fluctuations have been studied extensively [1, 6 . Recently McNamara et al. 7. carried out a careful analysis of volume histograms sampled over time. However, if the systems under study are not ergodic - an a priori assumption in most of these studies - then time averages over fluctuations for one system need not be the same as averages over the initial conditions. To tackle this problem, one should ideally study an ensemble of systems prepared in the same macroscopic way and follow the evolution of their volume with time. To our knowledge, such a study is still lacking.

In this paper, we use numerical simulations to study the evolution of an ensemble of granular systems prepared with the same macroscopic protocol. We analyse the rate of convergence of these histograms and find that, under certain conditions, individual trajectories are nonergodic. Finally, we find that our results are incompatible with Edwards' hypothesis, namely, that the dependence of the volume statistics on preparation protocol can be captured by a simple Boltzmann weight [8].

\section{II. "EQUILIBRIUM" DISTRIBUTION}

\section{A. Numerical simulation}

The shaking experiment is simulated using a dissipative, event-driven MD simulation of frictionless hard spheres subject to gravity. We use lateral periodic boundary conditions and place a wall at the bottom of the simulation box.

A known problem with event-driven schemes applied to dissipative systems is that a linear restitution coefficient makes the simulation "freeze" locally in higher-density regions [9]. To avoid this problem, we use a nonlinear restitution coefficient, so that the spheres do not loose all their kinetic energy, even after many collisions. When the system relaxes after an external perturbation (e.g. a single tap), the final state will have a well-defined structure, but the spheres will still move locally-albeit with a typical displacement amplitude that is much smaller than their size. We assume that the packings thus obtained are representative of the typical stable configurations that are obtained in real experiments at sufficiently high densities.

We start every simulation by generating an equilibrated fluid configuration of 1000 hard spheres at packing fraction $\phi=0.2$ in a cubic simulation box. We then switch on gravity and let the system reach mechanical equilibrium, as explained above. This is the preparation stage of the simulation. Once the packing has settled, we "tap" the system by accelerating the bottom layer of the spheres impulsively. Concretely, we add to these spheres the vertical velocity $A \sqrt{g a}$, where $A$ is a dimensionless tapping amplitude, $g$ is the acceleration of gravity, and $a$ is the diameter of the spheres. We repeat this process at regular time intervals, which are longer than the typical time it takes the packing to settle after a perturbation. 


\section{B. Volume histogram evolution}

To study the ensemble statistics of our system, we prepare about 50000 stable initial conditions as described above. The corresponding distribution of the initial volumes is denoted $\rho_{0}(V)$. For a given amplitude $A$, the volume histogram after $i$ tapping steps is denoted $\rho_{i}^{A}(V)$. The volume of a packing is defined as the smallest axisaligned cuboidal volume that completely contains all the spheres, and whose bottom face lies in the plane of the wall.

In analogy with the Gibbs approach in statistical mechanics, we define the statistical "equilibrium" ensemble distribution as the asymptotic volume distribution obtained when the number of taps tends to infinity. If there is a well-defined time-scale for approach to the steady state, then this procedure should yield a good approximation of the invariant distribution.

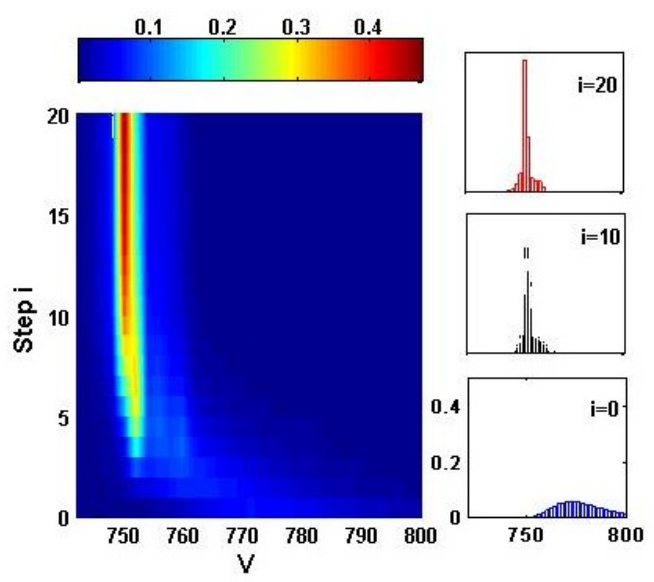

FIG. 1. Typical volume histogram evolution for an ensemble of about 50000 replicas of a system of 1000 spheres. The picture shown corresponds to an amplitude $A$, equal to 6 .

In Fig, 1 we show the evolution of a typical volume histogram as a function of the number of tapping steps. Starting from a very broad volume distribution, the ensemble histograms become narrower, and eventually reach an invariant shape.

\section{Convergence analysis}

To quantify the convergence oberved for the volume histograms in Fig.1. we want to assess to what extent a sequence $\left\{\rho_{i}^{A}(V)\right\}_{i=1,2, .}$ becomes independent of $i$ for large $i$. For this purpose, we introduce the 2sample Kolmogorov-Smirnov (KS) statistic $D\left[\rho_{1}, \rho_{2}\right]$ between two one-dimensional histograms $\rho_{1}$ and $\rho_{2}$ [10]:

$$
D\left[\rho_{1}, \rho_{2}\right] \equiv \sup _{V}\left\{\left|F_{1}(V)-F_{2}(V)\right|\right\},
$$

where $F_{1}$ and $F_{2}$ are the respective cumulative distribution functions associated with histograms $\rho_{1}$ and $\rho_{2}$.

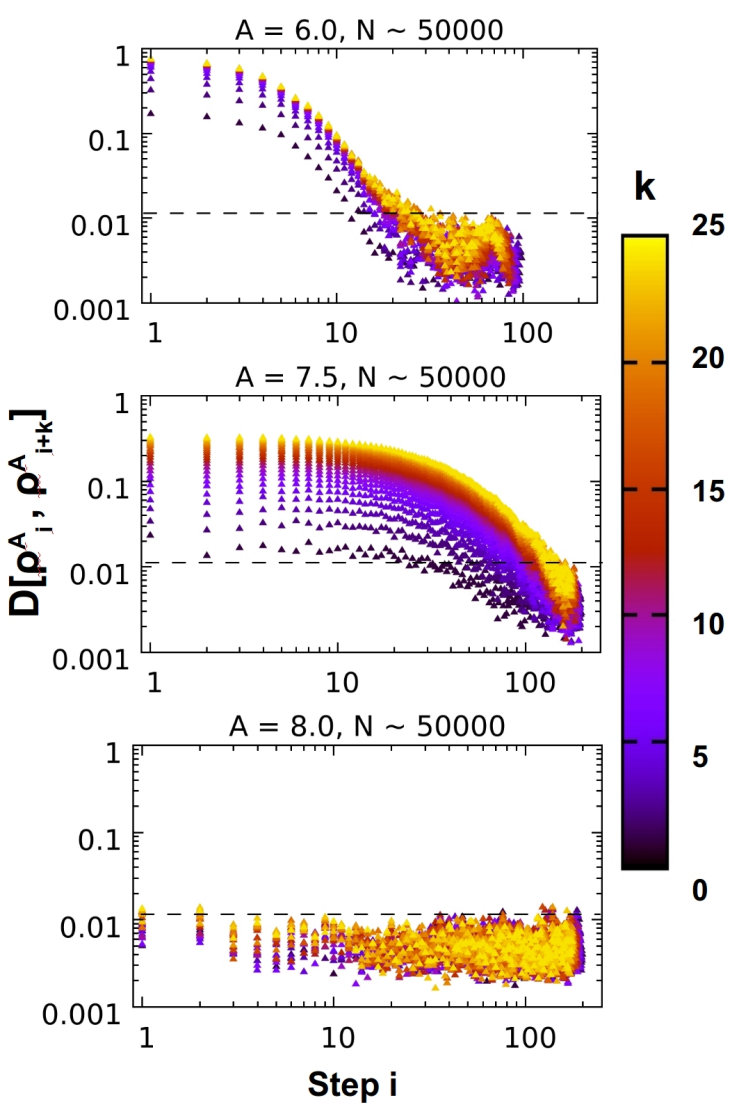

FIG. 2. Convergence analysis. Plot of the KS distances $D\left[\rho_{i}^{A},{ }_{i+k}^{A}\right]$ ( $k$ running from 1 to 25 being represented by the different colors) for different typical amplitudes corresponding to different relaxation regimes: $A=6.0$ (top), $A=7.5$ (middle) and $A=8.0$ (bottom). The dashed line in each plot corresponds to the critical value $\epsilon^{*}$ for histograms built from 50000 data points.

This "distance" is commonly used as a test for the null hypothesis that the two histograms $\rho_{1}$ and $\rho_{2}$ are different realisations of the same underlying distribution. This hypothesis can be rejected with $99 \%$ of certainty if [1]:

$$
D\left[\rho_{1}, \rho_{2}\right]>1.63 \sqrt{\frac{n_{1}+n_{2}}{n_{1} n_{2}}} \equiv \epsilon^{*}\left(n_{1}, n_{2}\right),
$$

where $n_{1}$ and $n_{2}$ are the respective number of samples used to build $\rho_{1}$ and $\rho_{2}$. From Eq.(2), we expect that the KS statistic will decrease as the accuracy of each histogram increases (i.e., $\epsilon^{*}\left(n_{1}, n_{2}\right)$ decreases), unless the two histograms sample different underlying distributions.

In practice, we seek the existence of an equilibrium step, denoted by $I(A)$, past which the deviation from the invariant histogram is within the statistical noise. In Fig, 2, we plot the KS distances $D\left[\rho_{i}^{A}, \rho_{i+k}^{A}\right]$ as a function of both $i$ (x-axis) and $k$ (color code). We also display the critical value $\epsilon^{*}=0.0109$ corresponding to histograms built from 50000 samples (dashed line, Eq.(2)). We identify $I(A)$ as the first step for which all colored points 
(from black for $k=1$ to bright yellow for $k=25$ ) are below the dashed line. We emphasize that it is not enough for $D\left[\rho_{i}^{A}, \rho_{i+1}^{A}\right]$ to be less than $\epsilon^{*}$. For example, as shown in Fig 2 in the case of $A=7.5$, this weaker condition is satisfied (black points) as early as the 30th tap, whereas our convergence criterion is fulfilled only after the step $I(7.5)=164$.

We also notice that the relaxation time increases when going from $A=6.0$ to $A=7.5$, whereas it decreases when going from $A=7.5$ to $A=8.0$.

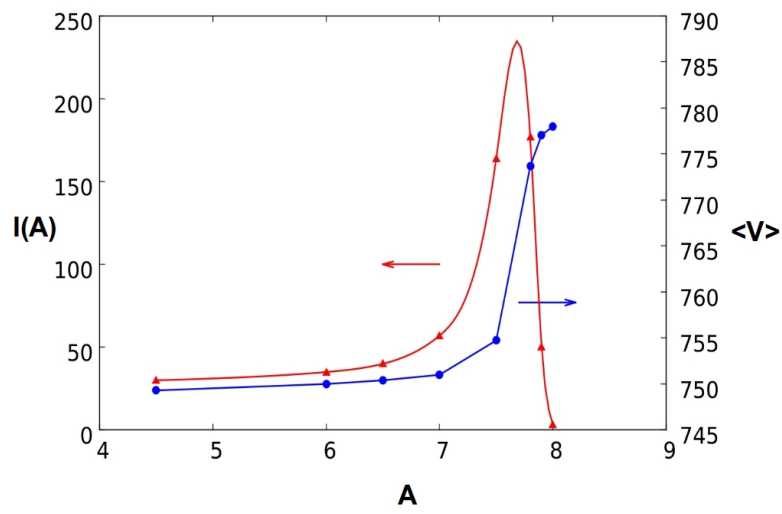

FIG. 3. Invariant properties. Red curve (triangles): relaxation number of steps $I(A)$ vs tapping amplitude, $A$. The line is a cubic spline interpolation, and is meant to guide the eye. Blue curve (circles): invariant average volume, $\langle V\rangle$, vs A.

In Fig, 3, we plot the mean invariant volumes, $\langle V\rangle$, and their corresponding equilibrium step, $I(A)$, for the amplitudes we have tested. We notice that $\langle V\rangle$ increases with tapping amplitude, as found in previous studies [1, 3, 5] 7]. The values of $I(A)$ are consistent with the number of steps required for the ensemble average volume to reach a plateau value for a given amplitude $A$ (data not shown). As stressed previously, we note that it is not an always decreasing function of $A$. To our knowledge, this is the first time that a nonmonotonic dependence of relaxation time scales on tapping amplitude has been observed. Normally, one would expect a decrease of the relaxation time as the tapping amplitude is increased [1, 3, 5,7], although a nonmonotonic dependence on $A$ was already suggested in Ref. [6] but in a different context. Our current understanding of this dependence is the following. At sufficiently low $A$, the ensemble behaves like a quenched glass. Within this regime, higher amplitudes result in the system exploring a larger local basin, which increases the time to sample the basin and, hence, the relaxation time. On the other hand, if we were to tap on the system strongly enough so that each shaking step is akin to our preparation protocol, then we should not observe any change in the ensemble histogram between successive taps, which would yield a vanishing relaxation time. These two limits can only be reconciled if the relaxation time depends nonmonotonically on tapping amplitude, as shown in Figs. 2 and 3.

\section{TESTS ON THE INVARIANT HISTOGRAMS}

A. Ergodicity analysis

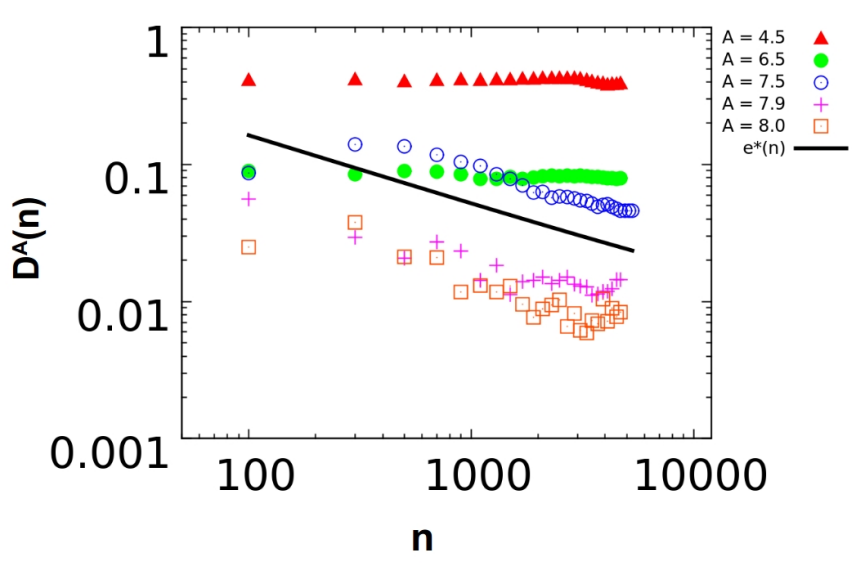

FIG. 4. Ergodicity analysis. Plot of the distance $D^{A}(n)$ as a function of the number of uncorrelated volume values, $n$, for different amplitudes, $A$. The plain black line corresponds to the threshold value $\epsilon^{*}(n)$ calculated from Eq. 2). The sampling is done after having tapped 200 times on the system.

To explain the behaviour observed in Figs. 2 and 3 , we have proposed in the preceding section that some conditions lead to nonergodicity. In this section, we test this proposal quantitatively.

Denote by $\rho_{n}^{A}(V)$ a volume histogram built from $n$ uncorrelated volume values taken from a very long trajectory of one system tapped with an amplitude $A$. In practice, we consider a single trajectory for a system tapped about $10^{5}$ times and we compute the corresponding volume correlation function. We then use the latter to select $n$ uncorrelated values belonging to this trajectory. Under the ergodic hypothesis, this "time" histogram and the invariant ensemble histogram, $\rho_{i>I(A)}^{A}(V)$, both sample the same underlying distribution. To test this idea, we again use the KS statistic. Let $D^{A}(n)$ be the quantity $D\left[\rho_{n}^{A}(V), \rho_{i>I(A)}^{A}(V)\right]$. If $D^{A}(n)$ is bigger than the rejection value $\epsilon^{*}(n)$ calculated from Eq. (2), with $n_{1}=n$ and $n_{2}=50000$, then the ergodic hypothesis is rejected. Recall that the KS statistic decreases with increasing $n$ if and only if the null hypothesis, ergodicity, is true. Otherwise, $D^{A}(n)$ saturates at a finite value when $n$ is large enough. Fig 4 shows that for high values of $n, D^{A}(n)$ indeed tends to saturate. In that case, the difference be- 
tween the saturation value of $D^{A}(n)$ and the curve $\epsilon^{*}(n)$ at a fixed $n$ serves as a useful measure of nonergodicity that we will call the ergodicity gap. Fig 4 shows that for tapping amplitudes $A=4.5,6.5$ and 7.5, trajectories are nonergodic, with ergodicty gaps that increase with decreasing tapping amplitude. In contrast, for $A=7.9$ and 8.0, trajectories are ergodic according to our criterion. These findings are consistent with our current understanding of the nonmonotonic trend for $I(A)$ observed in Figs. 2 and 3

\section{B. Compatibility with Edwards' prior}

In 1989, Edwards et al. 8 suggested that if a granular material is submitted to a protocol that yields different packing volumes then the probability of occurence of a given stable configuration is proportional to a Boltzmann weight $e^{-\beta V}$, where $V$ is the actual volume of the packing configuration. Since then, many studies have tested this idea and its consequences, but no consensus has been reached 3, 7, 12, 17.

In their study, McNamara et al. tested Edwards' prior by generating time-sampled volume histograms for different tapping amplitudes and looking directly at ratios between between pairs of histograms. This procedure is known as the overlapping histogram method [18: if the logarithm of the ratio between two histograms is linear, then there is a potential compatibility with Edwards' canonical assumption. Otherwise, this assumption should be rejected, at least for the tested protocol. McNamara et al. found a linear behaviour for the log ratios, for the amplitudes that they tested both numerically and experimentally. Here, we apply the same test on our own invariant ensemble histograms.

Fig 5 shows the log ratio of two invariant ensemble histograms ( $A=7.5$ with $A=8.0$ for the main plot and $A=4.5$ with $A=6.5$ in the inset). We find that over a limited range of volumes where the histograms overlap significantly, there is indeed a linear decrease in the log ratio of the two invariant histograms, consistent with the findings in Ref. 7]. However, we also find that for high volumes, this ratio tends to saturate, whereas for low volumes, it rapidly decreases. These trends are present for all pairs of invariant volume histograms that we have tested, including those where the two histograms are apparently "closeby".

It is worth noting that the authors of Ref. 7] have already suggested the possibility that the observed linearity is only true locally. Moreover a close look at their own figures reveals deviations from linearity for very high and very low volumes that are consistent with our own observations (Fig 5 ).

Overall, Edwards' canonical hypothesis as a global property is not compatible with the protocol we are testing. Although this is not the first time that a strong disagreement with Edwards' theory has been found [19], to our knowledge, this is the first time that a full his-

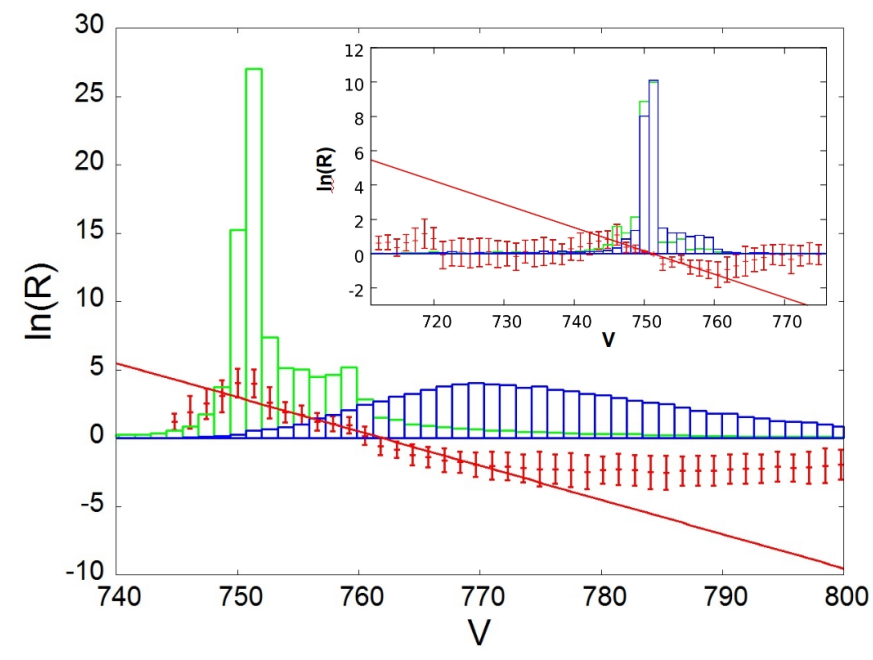

FIG. 5. Overlapping Histograms. The red points correspond to the plot of the logarithm of the ratios $\rho_{i>I_{7.5}}^{7.5} / \rho_{i>I_{8.0}}^{8.0}$ (main figure) and $\rho_{i>I_{4.5}}^{4.5} / \rho_{i>I_{6.5}}^{6.5}$ (inset). We superimposed the corresponding invariant histograms in both the main figure and the inset. The histogram in green always corresponds to the smallest amplitude in a given pair. The plain lines are the best linear fits through the midpoints within the crossover regions.

togram analysis reports incompatibility with Edwards' canonical assumption for vibrated granular matter. Our study does not rule out the possibility of a local compatibility with Edwards' assumption but this is already different from Edwards' orignal theory.

\section{CONCLUSION}

In this letter, we introduced an ensemble volume satistic for a simulated vertically vibrated granular system. To quantitatively assess the properties of the generated sequences of ensemble histograms, we used the KS test. This allowed us to devise a convergence criterion for a sequence of histograms. Subsequently, we tested the ergodicity of a tapped system as a function of the tapping amplitude, $A$, and found clear evidence for nonergodicity when the tapping amplitude is low. Finally, we tested the compatibility of our invariant histograms with Edwards' hypothesis and concluded that it is not compatible with our simulation protocol.

We should point out that the results found in this paper depend a priori on the chosen tapping protocol and also on the preparation stage. Although the dependence of our findings on the preparation protocol is hard to predict, the tools that we introduced to characterize our ensemble histograms can be used to test any numerical or experimental protocol. 


\section{ACKNOWLEDGMENTS}

Fabien Paillusson is grateful to Nicolas Dorsaz, Frank Smallenburg and Patrick Varilly for very helpful dis- cussions. This work has been supported by the EPSRC grant $N^{\circ} \mathrm{EP} / \mathrm{I} 000844 / 1$. D.F. acknowledges support from ERC Advanced Grant 227758, Wolfson Merit Award 2007/R3 of the Royal Society of London.
[1] N. Mueggenburg, Phys.Rev.E 85, 041305 (2012)

[2] M. Pica Ciamarra, M. Nicodemi, and A. Coniglio, Phys. Rev. E 75, 021303 (2007)

[3] E. Nowak, J. Knight, E. Ben-Naïm, H. Jaeger, and S. Nagel, Phys. Rev. E 57, 1971 (1998)

[4] A. Yu, X. An, R. P. Zou, R. Yang, and K. Kendall, Phys. Rev. Lett. 97, 265501 (2006)

[5] L. A. Pugnaloni, M. Mizrahi, C. Carlevaro, and F. Vericat, Phys.Rev.E 78, 051305 (2008)

[6] J. Brey and A. Prados, Phys.Rev.E 63, 061301 (2001)

[7] S. McNamara, P. Richard, S. Kiesgen de Richter, G. Le Caër, and R. Delannay, Phys. Rev. E 80, 031301 (2009)

[8] S. Edwards and R. Oakeshott, Physica A 157, 1080 (1989)

[9] I. Goldhirsch and G. Zanetti, Phys.Rev.Lett. 70, 1619 (1993)

[10] W. Press, S. Teukolsky, W. Vetterling, and B. Flannery, Numerical Recipes in $C$, second edition (Cambridge Uni- versity Press, 1992)

[11] N. Smirnov, Ann. Math. Statist 19, 279 (1948)

[12] R. Blumenfeld and S. F. Edwards, J. Phys. Chem. B 113, $3981(2009)$

[13] S. Henkes and B. Chakraborty, Phys. Rev. E 79, 061301 (2009)

[14] R. K. Bowles and S. S. Ashwin, Phys. Rev. E 83, 031302 (2011)

[15] T. Aste and T. Di Matteo, Phys. Rev. E 77, 021309 (2008)

[16] K. Wang, C. Song, P. Wang, and H. Makse, EPL 91, 68001 (2010)

[17] F. Lechenault and K. E. Daniels, Soft Matter 6, 3074 (2010)

[18] C. Bennett, J.Comp.Phys. 22, 245 (1976)

[19] G.-J. Gao, J. Blawzdziewicz, and C. S. O'Hern, Phys. Rev. E 74, 061304 (2006) 